\title{
Quantitative Calculation of the Spatial Extension of the Kondo Cloud
}

\author{
Gerd Bergmann \\ Department of Physics \\ University of Southern California \\ Los Angeles, California 90089-0484 \\ e-mail: bergmann@usc.edu
}

November 1, 2018

\begin{abstract}
The internal s-electron polarization in the ground state of a Friedel-Anderson and a Kondo impurity is calculated. The Wilson basis with exponentially fine energies close to the Fermi level is used and expressed in terms of free electron waves with linear energy-momentum dispersion. It is found that in the singlet state the magnetic moment of the d-electron is screened by an s-electron cloud. The linear extension of the cloud is inversely proportional to the Kondo energy. When the singlet state is destroyed the polarization cloud is absent.
\end{abstract}




\section{Introduction}

The properties of magnetic impurities in a metal is one of the most intensively studied problems in solid state physics. The work of Friedel [1] and Anderson [2] laid the foundation to understand why some transition-metal impurities form a local magnetic moment while others don't. Kondo [3] showed that multiple scattering of conduction electrons by a magnetic impurity yields a divergent contribution to the resistance in perturbation theory. Kondo's paper stimulated a large body of theoretical and experimental work which changed our understanding of d-and f-impurities completely (see for example [4], [5], 6], 7], 8], [9], [10], [11], [12], [13]). A large number of sophisticated methods were applied in the following three decades to better understand and solve the Kondo and Friedel-Anderson problems. In particular, it was shown that at zero temperature the Friedel-Anderson impurity is in a non-magnetic state. To name a few of these methods: scaling [14, renormalization [15], [16], [17], [18] Fermi-liquid theory [19], [20], slave-bosons (see for example [21]), large-spin limit [22], [23]. After decades of research exact solutions of the Kondo and Friedel-Anderson impurities were derived with help of the Bethe-ansatz [24], [25], [26], representing a magnificent theoretical achievement. The experimental and theoretical progress has been collected in a large number of review articles [7], [8], [9], [10], [11], [12], [13], [15], [20], [21], [23], [24], [25], 26], 27].

One of the most controversial aspects of the Kondo ground state is the so-called Kondo cloud within the radius $\xi_{K}$ where $\xi_{K}$ is called the Kondo length

$$
\xi_{K}=\frac{\hbar v_{F}}{k_{B} T_{K}}
$$

( $k_{B} T_{K}=$ Kondo energy, $v_{F}=$ Fermi velocity of the s-electrons).

The idea is to divide the ground state $\Psi_{K}$ of a Kondo impurity into two parts with opposite d-spins. (By reversing all spins one can transform one component into the other one.) The proponents of the Kondo cloud argue that in each component there is an s-electron within the Kondo sphere which compensates the d-spin. This s-electron forms a singlet state with the d-spin. An important assumption of the Kondo-cloud proponents is that, above the Kondo temperature, the bond is broken and this screening cloud evaporates from the Kondo sphere.

Already in the 1970's there were a number of theoretical papers with different predictions about the Kondo cloud which stimulated several experimental investigations. In most of the experiments nuclear magnetic resonance (NMR) was used, for example in $\mathrm{Cu}$ samples with dilute Fe-Kondo impurities. (For details see ref. 28] which contains also an overview of the theoretical predictions at that time). By applying a magnetic field (small enough so that it does not destroy the Kondo singlet state) NMR was used to measure the electron spin polarization at shells of $\mathrm{Cu}$ atoms around the Fe impurity. The line shift (adjusted with the temperature dependent susceptibility of the Fe impurity) did not show any change when 
the temperature crossed the Kondo temperature. This contradicted the concept that, in the Kondo ground state, the d-impurity was paired with and screened by an s-electron which evaporates above the Kondo temperature.

In recent theoretical papers the argument is made that an NMR experiment cannot possibly detect the screening electron because of the large volume of the Kondo sphere. With a radius of the order of $0.1 \mu \mathrm{m}$ (or larger) the Kondo sphere contains more than $2.0 \times 10^{8}$ atoms. Then the Kondo cloud is only a faint Kondo fog. It is spread so thinly that the change of polarization felt by an individual $\mathrm{Cu}$ atom can not be detected in an NMR experiment.

Actually the experimental side has not improved so far but a number of theoretical suggestions have been published since 2000 which propose to observe the Kondo cloud in sub-micron structures, in particular in connection with quantum dots [29], [30], [31], [32], [33]. A number of these papers also contain numerical renormalization calculation to obtain the correlation functions between the impurity spin and the spin of the conduction electrons.

In this paper I calculate quantitatively the screening cloud of a Friedel-Anderson and a Kondo impurity in the magnetic and the singlet state.

The paper is organized as follows. In section 2 the theoretical background of this paper will be introduced, the FAIR method. In section 3 the numerical results for the spatial density of the s-electrons will be reported and discussed for several cases. First the spatial extent of the simple Friedel d-resonance with spinless electrons is discussed. Since the physics of the Friedel impurity is simple this consideration will give us confidence in the use of the Wilson wave functions. In the next step the electronic environment of the Friedel-Anderson (FA) impurity is investigated. The calculation is first performed for its magnetic state. The latter represents the magnetic building block of the singlet ground state of the FA-impurity which is investigated next. Last, but not least, the ground state of the Kondo Hamiltonian is analyzed. The subtle modification between the magnetic and the singlet ground state is discussed. Section 4 contains the conclusion. In the appendix details of the FAIR states and the Wilson states are presented, including the wave functions of the Wilson states and their density in real space.

\section{Theoretical Background}

\subsection{The FAIR Method}

To investigate the question of a Kondo cloud I first consider a Friedel-Anderson impurity with spin $1 / 2$ in a metal host. The underlying Hamiltonian consists of two Friedel resonance Hamiltonians (one for each spin) and a Coulomb term $U n_{d, \uparrow} n_{d, \downarrow}$.

$$
H_{F A}=\sum_{\sigma}\left\{\sum_{\nu=1}^{N} \varepsilon_{\nu} c_{\nu \sigma}^{\dagger} c_{\nu \sigma}+E_{d} d_{\sigma}^{\dagger} d_{\sigma}+\sum_{\nu=1}^{N} V_{s d}(\nu)\left[d_{\sigma}^{\dagger} c_{\nu \sigma}+c_{\nu \sigma}^{\dagger} d_{\sigma}\right]\right\}+U n_{d, \uparrow} n_{d, \downarrow}
$$


Here $c_{\nu \sigma}^{\dagger}$ represent the creation operators of the Wilson s-electron state (which are discussed below), $d_{\sigma}^{\dagger}$ represents the creation operator of the d-state at the impurity and $n_{d, \sigma}$ are the operators of the d-occupation.

In the appendix I briefly describe the development of a very compact solution for the magnetic state and the singlet state. It uses two localized s-states $a_{0+}^{\dagger}$ and $a_{0-}^{\dagger}$ as artificial Friedel resonance states or FAIR states (Friedel Artificially Inserted Resonance states). The ground state solutions in terms of the FAIR basis have been studied in recent years for the Friedel Hamiltonian [34], [35, the magnetic and the singlet state of the Friedel-Anderson Hamiltonian [36], 37] and the Kondo Hamiltonian [38]. The compact and explicit form makes these solutions ideal for calculation of spatial properties. The compact ground states which are used in this paper are given for the Friedel impurity by equ. (5), the magnetic state by equ. (3), the singlet state of the FA impurity by equ. (4) and the Kondo ground state by equ. (6) .

The potentially magnetic state $\Psi_{M S}$ of the Friedel-Anderson Hamiltonian (which is the building block for the singlet state $\Psi_{S S}$ ) has the following form

$$
\Psi_{M S}=\left[A_{s, s} a_{0-\downarrow}^{*} a_{0+\uparrow}^{*}+A_{d, s} d_{\downarrow}^{*} a_{0+\uparrow}^{*}+A_{s, d} a_{0-\downarrow}^{*} d_{\uparrow}^{*}+A_{d, d} d_{\downarrow}^{*} d_{\uparrow}^{*}\right] \prod_{i=1}^{n-1} a_{i-\downarrow}^{*} \prod_{i=1}^{n-1} a_{i+\uparrow}^{*} \Phi_{0}
$$

The magnetic state $\Psi_{M S}$ has the same form as the mean field solution $\Psi_{m f}$. The only difference is that the state $\Psi_{M S}$ opens a wide playing field for optimization: (i) The FAIR states $a_{0+}^{\dagger}$ and $a_{0-}^{\dagger}$ can be individually optimized, each one defining a whole basis $\left\{a_{i \pm}^{\dagger}\right\}$ (which yields a Hamiltonian of the form in Fig.10a in the appendix) and (ii) the coefficients $A_{\alpha, \beta}$ can be optimized as well, fulfilling only the normalization condition $A_{s, s}^{2}+A_{d, s}^{2}+A_{s, d}^{2}+$ $A_{d, d}^{2}=1$. This yields a good treatment of the correlation effects. The optimization procedure is described in detail in ref. [36]. The state is denoted as the (potentially) magnetic state $\Psi_{M S}$. It is expected to be a good solution above the Kondo temperature.

The Coulomb interaction in the Friedel-Anderson impurity destroys the symmetry between the occupation of spin up and down electrons and generates a magnetic moment (if $U$ is large enough). However, this state with broken symmetry is degenerate. Its counterpart with reversed spins has the same energy, and at zero temperature the two form a new symmetric state which has a different symmetry than original one. This is somewhat analogous to an atom in a harmonic potential. If some process transforms the harmonic potential into a double well potential the symmetry is broken. Although the atom has now an energy minimum on the left or right side its atomic wave function creates a new symmetric state by forming a symmetric superposition of left and right occupation.

In a similar way I use the magnetic state as a building block for the singlet ground state. $\Psi_{M S}$ together with its counterpart where all spins are reversed yield two states $\bar{\Psi}_{M S}(\uparrow \downarrow)$ and $\overline{\bar{\Psi}}_{M S}(\downarrow \uparrow)$. The singlet ground state is then given by

$$
\Psi_{S S}=\bar{\Psi}_{M S}(\uparrow \downarrow) \pm \overline{\bar{\Psi}}_{M S}(\downarrow \uparrow)
$$




$$
\begin{aligned}
\Psi_{S S} & =\left[\overline{A_{s, s}} a_{0-\downarrow}^{*} a_{0+\uparrow}^{*}+\overline{A_{d, s}} d_{\downarrow}^{*} a_{0+\uparrow}^{*}+\overline{A_{s, d}} a_{0-\downarrow}^{*} d_{\uparrow}^{*}+\overline{A_{d, d}} d_{\downarrow}^{*} d_{\uparrow}^{*}\right] \prod_{i=1}^{n-1} a_{i-\downarrow}^{*} \prod_{i=1}^{n-1} a_{i+\uparrow}^{*} \Phi_{0} \\
& +\left[\overline{\overline{A_{s, s}}} a_{0+\downarrow}^{*} a_{0-\uparrow}^{*}+\overline{\overline{A_{d, s}}} a_{0+\downarrow}^{*} d_{\uparrow}^{*}+\overline{\overline{A_{s, d}}} d_{\downarrow}^{*} a_{0-\uparrow}^{*}+\overline{\overline{A_{d, d}}} d_{\downarrow}^{*} d_{\uparrow}^{*}\right] \prod_{i=1}^{n-1} a_{i+\downarrow}^{*} \prod_{i=1}^{n-1} a_{i-\uparrow}^{*} \Phi_{0}
\end{aligned}
$$

(If all spin up states are moved to the left the plus sign applies). The bars on top of $\Psi_{M S}$ are supposed to point out that all the parameters such as $a_{0 \pm}^{\dagger}$ and $A_{\alpha, \beta}$ have to be newly optimized.

The resulting ground state is a very good approximation for the exact ground state. Its ground-state energy and the d-occupations for zero, one and two d-electrons at the impurity are of the same quality as the best numerical calculations in the field [39].

In the numerical construction of the FAIR ground state the states $a_{0 \sigma}^{\dagger}$ is rotated in the $N$-dimensional Hilbert space until the energy expectation value reaches its minimum. Each time one has to construct the other states of the basis $\left\{a_{i \sigma}^{\dagger}\right\}$ so that they are orthonormal and non-interacting. This cannot be done with $10^{23}$ electron states. Therefore we follow Wilson in constructing a reduced basis, in our case with generally $N=50$ Wilson states. The definition of the Wilson states, their properties and in particular their wave function in real space is discussed in the appendix.

\section{$3 \quad$ Numerical Results}

\subsection{Friedel Impurity}

I start the analysis with the Friedel resonance of a spinless d-impurity. Since the Friedel Hamiltonian is a single particle Hamiltonian which can be solved exactly, one is here on familiar ground and the interpretation of the results will guide us in the less known territory of the Friedel-Anderson and the Kondo impurity.

Given is the $n$-particle free electron state $\Psi_{0}^{(n)}=\prod_{\nu=0}^{n-1} c_{\nu}^{\dagger} \Phi_{0}$ in terms of Wilson states. A (spinless) Friedel d-impurity is introduced at the position $x=0$. This impurity has an s-dinteraction with the states $\psi_{\nu}(x)=\left\langle c_{\nu}^{\dagger} \Phi_{0} \mid x\right\rangle$. Due to the impurity the $n$-electron state $\Psi_{0}^{(n)}$ is modified. As discussed in the appendix the exact ground state of the Friedel impurity can be written as

$$
\Psi_{F}=\left(A a_{0}^{\dagger}+B d^{\dagger}\right) \prod_{i=1}^{n-1} a_{i}^{\dagger} \Phi_{0}
$$

The composition of the FAIR state $a_{0}^{\dagger}$ and the coefficients $A, B$ are obtained by an iteration process in which the FAIR state is rotated in Hilbert space into its optimal orientation. Details of this iteration process and the construction of the full ground state have been described in previous papers [34], [37].

In the next step we have to calculate the integrated electron density in the presence of the Friedel d-impurity at the origin. This calculation is discussed in the appendix. The 
density of the state $\psi_{\nu}(x)$ is given by

$$
\rho_{\nu}^{0}(x)=\left|\psi_{\nu}(x)\right|^{2}=2^{\nu+3} \frac{\sin ^{2}\left(\pi x \frac{1}{2^{\nu+2}}\right)}{\pi x} d x
$$

for $\nu<N / 2$. In the evaluation we use $N=50$ Wilson states. From the above equation one recognizes that the density $\rho_{\nu}^{0}$ of the state $\psi_{\nu}$ lies roughly in the interval between $2^{\nu-2}$ and $2^{\nu+2}$ (in units of $\lambda_{F} / 2$ ). Since for negative energies $\nu$ takes the values from 0 to $(N / 2-1$ ) the different wave functions $\psi_{\nu}(x)$ have very different spatial ranges and therefore very different densities, the lowest being of the order of $2^{-25}<3 \times 10^{-8}$. Therefore one has to be careful in the summation of the different contributions.

In the numerical evaluation we calculate (i) the s-electron density in the presence and absence of the d-impurity, (ii) form the net s-electron density $\rho(x)$ as the difference, (iii) integrate the net s-electron density from $x=0$ to $r$ where $r$ is increased on an exponential scale, $r=2^{l}$ and plot the net integrated s-electron density versus $l=\log _{2}(r)$. This net integrated density $q(r)=\int_{0}^{r} \rho(x) d x$ is plotted in Fig.1 as a function of the distance from the impurity. The full triangles and circles give the integrated net s-electron density for the two components $\Psi_{A}=\prod_{i=0}^{n-1} a_{i}^{\dagger} \Phi_{0}$ and $\Psi_{B}=d^{\dagger} \prod_{i=1}^{n-1} a_{i}^{\dagger} \Phi_{0}$ of the ground state, and the squares for the full ground state $\Psi_{F}$. The curve for $\Psi_{A}$ increases from 0 to 0.75 roughly between $l=-2$ and +3 . Around $l=23$ it drops back to zero. It has to return to zero because the total number of occupied states in $\Psi_{A}$ is the same as before in the state $\Psi_{0}=\prod_{i=0}^{n-1} c_{\nu}^{\dagger} \Phi_{0}$, i.e. equal to $N / 2$. The equivalent distance $r=2^{23}$ corresponds roughly to the maximum range of the wave functions. In a way it can be considered as the border or surface of the theoretical sample. Therefore the interpretation of this curve in Fig.1 is that in the state $\Psi_{A}$ a fraction of 0.75 electrons has moved from the surface towards the d-impurity. There it surrounds the empty d-state in the spatial region between $r=2^{-2}$ and $2^{3}$. (The component $\Psi_{A}$ has no d-electron). The behavior of the integrated net density beyond about $l \approx 20$ is a surface effect and of no interest for the present investigation. If one increases the number of cells from $N$ to $N^{\prime}$ then this part of the curve moves by $\left(N^{\prime}-N\right) / 2$ to the right while the left part of the curve remains unchanged.

The full circles in Fig.1 give the change of the net integrated density of s-electrons for the state $\Psi_{B}$ as a function of distance. At large distances $\left(r \approx 2^{23}\right)$ it approaches the value -1 because this state has only $(N / 2-1)$ s-electrons. Between the distances $l=-2$ and +3 the curve for $\Psi_{B}$ assumes the value -.25 . If one adds to the integrated s-electron density the one d-electron at the origin then one obtains the dotted curve which starts on the left at one and approaches zero at large distances. It is interesting to notice that in the range $5<l<20$ the total integrated density of d- and s-electrons is equal to 0.75 for both wave functions $\Psi_{A}$ and $\Psi_{B}$. This means both components have accumulated the same charge close to the impurity. As discussed below this corresponds to a Friedel phase shift of $\delta=0.75 \pi$.

In this specific example the parameters of $\left|V_{s d}^{0}\right|^{2}=0.1$ and $E_{d}=-.135$ were chosen so that the occupation numbers take the values $A^{2}=.25$ and $B^{2}=.75$. Then the net integrated 
density of $\Psi_{A}$ times the occupation number $A^{2}$ yields $0.75 * 0.25 \approx 0.188$ and just cancels the corresponding product of $0.75^{*}(-.25)$ for the state $\Psi_{B}$. As a result the total integrated density of s-electrons as a function of distance from the impurity vanishes as the curve for $\Psi_{F}$ shows (full squares). There is no change of s-electron density about the impurity. On the other hand the curve approaches the value of -.75 for large distance. This is the charge which has been moved from the surface into the d-state. This charge is predicted by the Friedel sum rule. The occupation $B^{2}=0.75$ of the state $\Psi_{B}$ means that the d-occupation is 0.75 , i.e. the phase shift is $\delta=.75 \pi$ and therefore the Friedel charge is $\delta / \pi=0.75$.

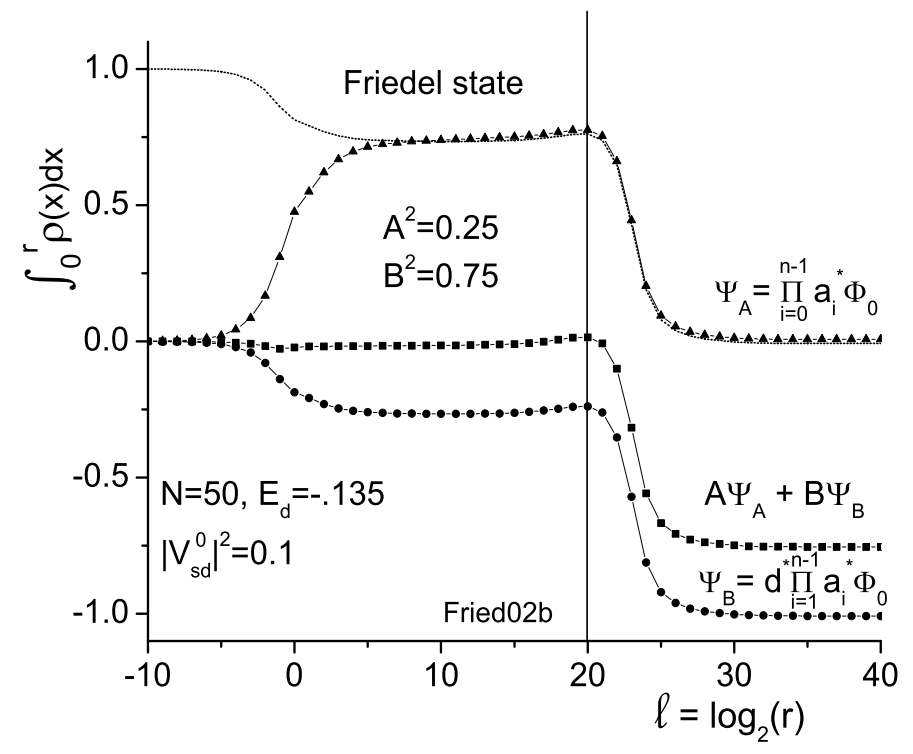

Fig.1: The net integrated density $\int_{0}^{r} \rho(x) d x$ is plotted versus the logarithm of the distance $l=\log _{2}(r)$ from the impurity for the components $\Psi_{A}, \Psi_{B}$ and the full wave function $\Psi_{F}$. For the dotted line the additional one d-electron has been added.

The Friedel resonance is also ideal testing ground for the spatial extension of the wave function. For that purpose the d-state is moved to the Fermi level $\left(E_{d}=0\right)$. The half width of the resonance is given by $\Delta=\pi\left|V_{s d}^{0}\right|^{2} \rho_{0}$ ( $\rho_{0}=$ density of states). This resonance width introduces an additional length scale into the problem. In the resonance the d-state hybridizes with the s-electrons in this energy range of $2 \Delta$. This corresponds to a wave number range of $k_{\Delta} \approx 2 \pi\left|V_{s d}^{0}\right|^{2} \rho_{0}$ and therefore a smearing of the wave function over a range $x_{\Delta} \propto$ $1 /\left(2 \pi\left|V_{s d}^{0}\right|^{2} \rho_{0}\right)\left(V_{s d}^{0}\right.$ is measured in unit of $\varepsilon_{F}$ and $x_{\Delta}$ in units $\left.\lambda_{F} / 2\right)$.

In the numerical evaluation I change the value of $\left|V_{s d}^{0}\right|^{2}$ in steps of two from 0.005 to 0.16 . Indeed one recognizes in Fig.2 that with decreasing $\left|V_{s d}^{0}\right|^{2}$ the increase of the integrated net density is stretched over an increasing length scale. Since the horizontal separation of the 
points by 1 corresponds to a factor of two in the length scale each curve is broader by a factor two than the previous one.

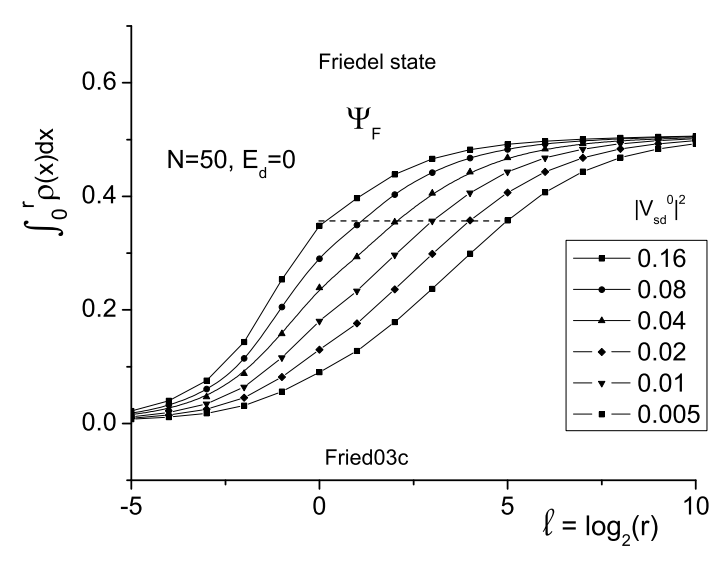

Fig.2: The net integrated density $\int_{0}^{r} \rho(x) d x$ for the state $\Psi_{A}=\prod_{i=0}^{n-1} a_{i}^{\dagger} \Phi_{0}$ for different values of $\left|V_{s d}^{0}\right|^{2}$ while the d-resonance lies at the Fermi level, $E_{d}=0$. The width of the wave function increases proportional to $1 / \pi\left|V_{s d}^{0}\right|^{2}$.

\subsection{The Magnetic State}

The magnetic state $\Psi_{M S}$ is the building block of the singlet state. Its multi-electron state is built from four Slater states and shown in equ. (3). I calculate the net integrated density of spin up and down, as well as total density and spin polarization in the vicinity of the impurity when it is in the magnetic state. For the present purpose I choose the parameters $E_{d}=0.5,\left|V_{s d}^{0}\right|^{2}=0.04, U=1$ and $N=50$. This yields a well developed magnetic moment of $\mu=0.93 \mu_{B}$. The occupation of the different components is $A_{s, s}^{2}=0.0294, A_{s, d}^{2}=0.0057$, $A_{d, s}^{2}=0.9355$ and $A_{d, d}^{2}=0.0294$.

In Fig. 3 the net densities are plotted. As discussed above, the range beyond $2^{20}$ corresponds to the rim or surface of the sample and is of no interest for the density distribution around the impurity. One recognizes that there is only a negligible polarization of the electron gas in the vicinity of the impurity. The important result of Fig.3 is that there is no 
polarization cloud around the magnetic state of the impurity.

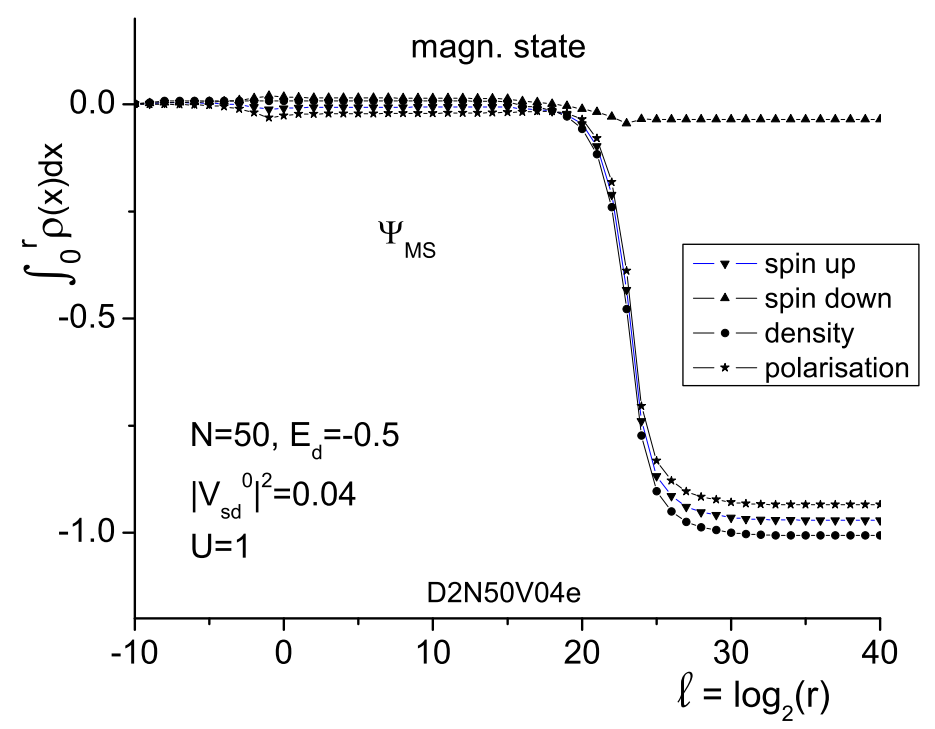

Fig.3: The net integrated density $\int_{0}^{r} \rho(x) d x$ of the s-electron within a distance $r$ from the impurity for spin up and down, as well as total density and spin polarization. The magnetic moment of the impurity is $0.93 \mu_{B}$.

\subsection{The Singlet State of the Friedel-Anderson Impurity}

The author derived the compact singlet ground state in ref. [37]. Its form is given in equ. (44). The singlet state does not have a net spin polarization because of the symmetry between spin up and down. However, the individual components may possess a spin polarization cloud. To investigate this question one has first to decide how one subdivides the symmetric state of the impurity. Since there are four Slater states which have either zero or two d-electrons one cannot divide the full state into two parts in which either the $d_{\uparrow \text { - }}$ or the $d_{\downarrow}$-state are occupied. A reasonable division would be into the states $\overline{\Psi_{M S}(\uparrow \downarrow)}$ and $\overline{\overline{\Psi_{M S}(\downarrow \uparrow)}}$. However, one may object that both states contain a component in which, for example, the $d_{\uparrow-s t a t e}$ is singly occupied. Nevertheless I use the components $\overline{\Psi_{M S}(\uparrow \downarrow)}$ as the magnetic component because in the singlet state the coefficients $\overline{A_{s, s}}, . . \overline{A_{d, d}}$ and $\overline{\overline{A_{s, s}}}, . . \overline{\overline{A_{d, d}}}$ have the same sign while in the triplet state they have opposite signs. (Their absolute values are in both cases pair-wise identical).

In the following plots in Fig.4 I choose the state $\overline{\Psi_{M S}(\uparrow \downarrow)}$ with the majority d-spin pointing up. The same parameters as for the magnetic state are used: $E_{d}=-0.5,\left|V_{s d}^{0}\right|^{2}=$ $0.04, U=1$. This yields the following coefficients: $A_{s s}^{2}=0.0146, A_{s d}^{2}=0.0028, A_{d s}^{2}=0.4629$ and $A_{d d}^{2}=0.0146$. They are about half the values of the magnetic state. Therefore on a superficial glance it appears that $\Psi_{s s} \approx(1 / \sqrt{2})\left[\Psi_{M S}(\uparrow \downarrow)+\Psi_{M S}(\downarrow \uparrow)\right]$. 
In Fig.4 the integrated densities of spin up and down electrons, their sum and difference (the polarization) are plotted versus the distance from the magnetic impurity (on a logarithmic scale). One recognizes that now one has considerable contributions to the integrated net densities of both spins. The polarization of the two contributions is no longer zero but reaches a value of -0.46 at a distance of $r \approx 2^{11.6}$. Since the magnetic state with net $\mathrm{d}$-spin up has only a weight of about $1 / 2$ it contributes an effective $d_{\uparrow}^{\dagger}$-moment of $0.93 / 2 \approx 0.46$. Therefore this d-spin is well compensated by the polarization of the s-electron background. The difference with the pure magnetic state is particularly striking. We observe a screening polarization cloud of s-electrons about the impurity within the range of $r \approx 2^{11.6}=3.1 \times 10^{3}$.

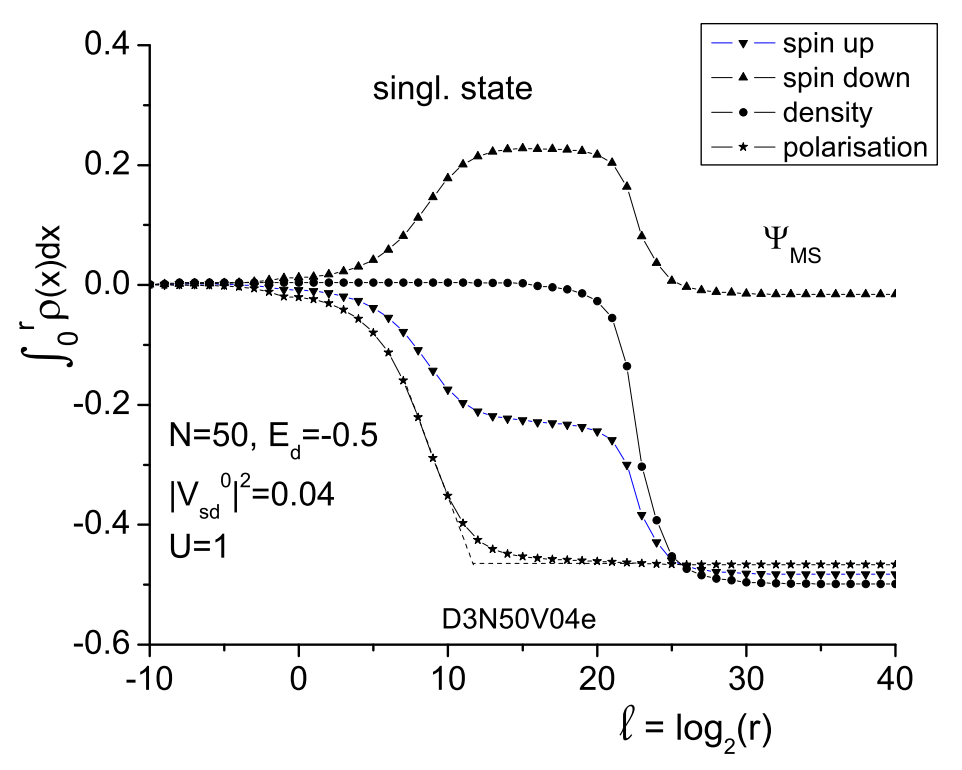

Fig.4: The net integrated density $\int_{0}^{r} \rho(x) d x$ within a distance $r=2^{l}$

from the d-spin up component of the impurity. Shown are the spin up, spin down components as well as the total density and the polarization. The $\mathrm{d}_{\uparrow}$-spin of $0.93 / 2$ is screened by 0.46 s-electrons within the range of $r \approx 2^{11.6}$.

The range of the screening cloud depends on the strength of $\left|V_{s d}^{0}\right|^{2}$. In Fig.5 a similar plot as in Fig.4 is performed for an s-d-coupling of $\left|V_{s d}^{0}\right|^{2}=0.1$. This time the polarization 
cloud extends only over a distance of $r \approx 2^{6.4} \approx 84$.

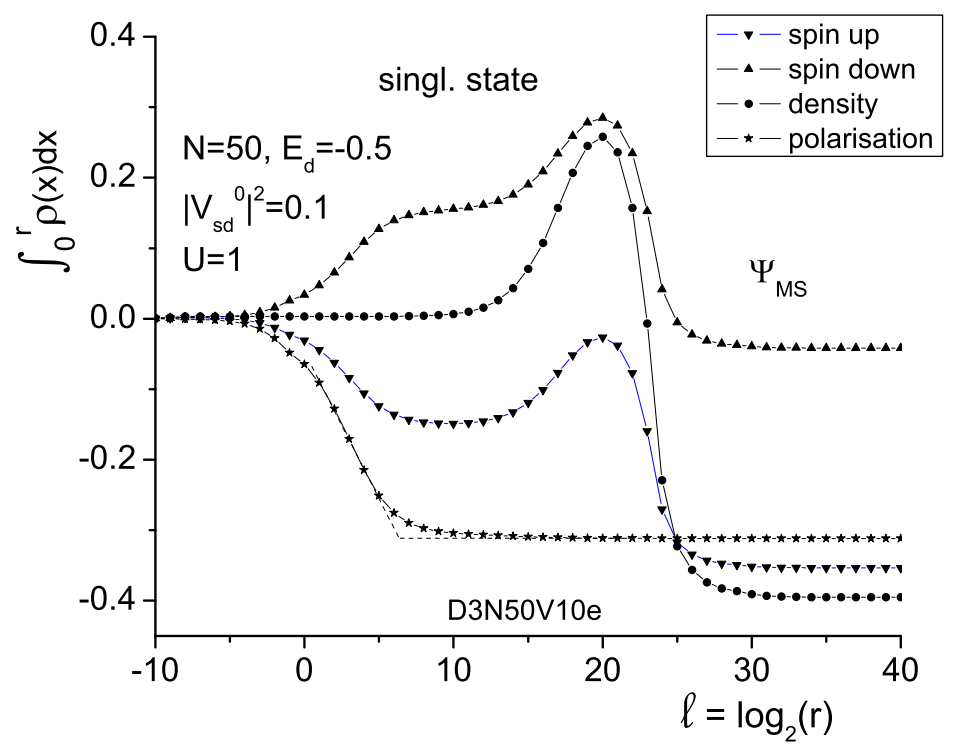

Fig.5: The net integrated density $\int_{0}^{r} \rho(x) d x$ within a distance $r=2^{l}$ form the d-spin up component of the impurity. Shown are the spin up, spin down components as well as the total density and the polarization. The $\mathrm{d}_{\uparrow}$-spin polarization cloud extends for $\left|V_{s d}^{0}\right|^{2}=0.1$ over a range of $r \approx 2^{6.4}$.

\subsection{The FAIR states}

It is remarkable how different the polarizations of the s-electrons in the magnetic state $\Psi_{M S}$ and the magnetic component of the singlet state $\overline{\Psi_{M S}}$ are. This is particularly surprising since the amplitudes $\overline{A_{\alpha, \beta}}=\overline{\overline{A_{\alpha, \beta}}}$ in the singlet state are roughly equal to $A_{\alpha, \beta} / \sqrt{2}$. However, in the singlet state one has a finite coupling between $\overline{\Psi_{M S}}$ and $\overline{\overline{\Psi_{M S}}}$. This affects the composition of the FAIR states $a_{0+}^{\dagger}$ and $a_{0-}^{\dagger}$. The two states $a_{0+}^{\dagger}$ and $a_{0-}^{\dagger}$ contain the whole information about the many electron states $\Psi_{M S}$ or $\Psi_{S S}$. When $a_{0 \pm}^{\dagger}$ are known the whole bases $\left\{a_{i+}^{\dagger}\right\}$ and $\left\{a_{i-}^{\dagger}\right\}$ and the coefficients $A_{\alpha, \beta}$ can be reconstructed. Therefore it is worthwhile to compare the two FAIR states for the magnetic and the singlet state.

For the magnetic state the coefficients $\alpha_{0 \pm}^{\nu}$ of the states $a_{0 \pm}^{\dagger}=\sum_{\nu} \alpha_{0 \pm} c_{\nu}^{\dagger}$ are plotted in Fig.6 versus the cell number $\nu$, which is a measure of the energy. While $a_{0+}^{\dagger}$ is essentially concentrated at positive energies the coefficients of $a_{0-}^{\dagger}$ have their main weight at negative energies. The two are mirror images with respect to the energy zero. 

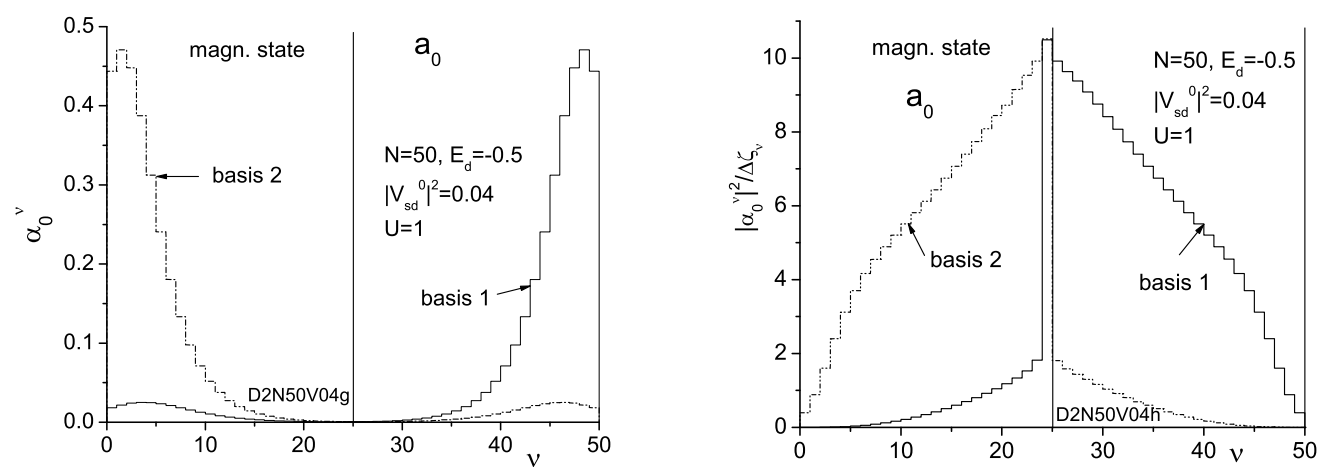

Fig.6: The coefficients $\alpha_{0 \pm}^{\nu}$ of the FAIR states $a_{0+}^{\dagger}$ and $a_{0-}^{\dagger}$ are plotted in terms of the states $c_{\nu}^{\dagger}$ for the magnetic state $\Psi_{M S}$. The full and the dashed curves represent $a_{0+}^{\dagger}$ and $a_{0-}^{\dagger}$ respectively.

Fig.6b: The density distribution of the $p_{\nu}=\left|\alpha_{0 \pm}^{\nu}\right|^{2} / \Delta_{\nu}$ as a function of as a function of $\nu$.

In Fig.6b the quantity $p_{\nu}=\left|\alpha_{0}^{\nu}\right|^{2} / \Delta_{\nu}$ is plotted as a function $\nu$, where $\Delta_{\nu}=\left(\zeta_{\nu+1}-\zeta_{\nu}\right)$ is the width of the energy cell $\mathfrak{C}_{\nu}$ of the Wilson state $\psi_{\nu}(x)$. The quantity $p_{\nu}$ is a function of the energy $p_{\nu}=p(\zeta)$, and $p(\zeta) d \zeta$ represents the weight of the original basis states $\varphi_{k}(x)$ in the energy window $(\zeta, \zeta+d \zeta)$ to the FAIR state $a_{0}^{\dagger}$. This weight is plotted in Fig.6b for the magnetic state as a function of $\nu$. It would be more natural to plot $p(\zeta)$ as a function of the energy. But for $\nu$ close to $N / 2$ the width of the energy cells $\mathfrak{C}_{\nu}$ is less than $10^{-6}$ and any dependence of $p(\zeta)$ on the energy cannot be resolved on a linear scale. The probability $p_{\nu}$ increases close to the Fermi energy. Again the contributions of $a_{0+}^{\dagger}$ and $a_{0-}^{\dagger}$ resemble mirror images.

In Fig.7a and Fig.7b the corresponding plots are shown for the magnetic component of the singlet state. While the overall shape of the coefficients in Fig.7a resembles that in Fig.6 the weight $p_{\nu \pm}=\left|\alpha_{0 \pm}^{\nu}\right|^{2} / \Delta_{\nu}$ close to the Fermi surface is very different for the singlet state and the magnetic state. While the two probabilities are mirror images for the magnetic case and reach a maximum value of about 10 one observes in the singlet state a maximum of about 400 and the weights $p_{\nu \pm}$ in $a_{0+}^{\dagger}$ and $a_{0-}^{\dagger}$ are essentially identical and not mirror images. The magnetic component of the singlet state is in a subtle way different from the 
magnetic state.
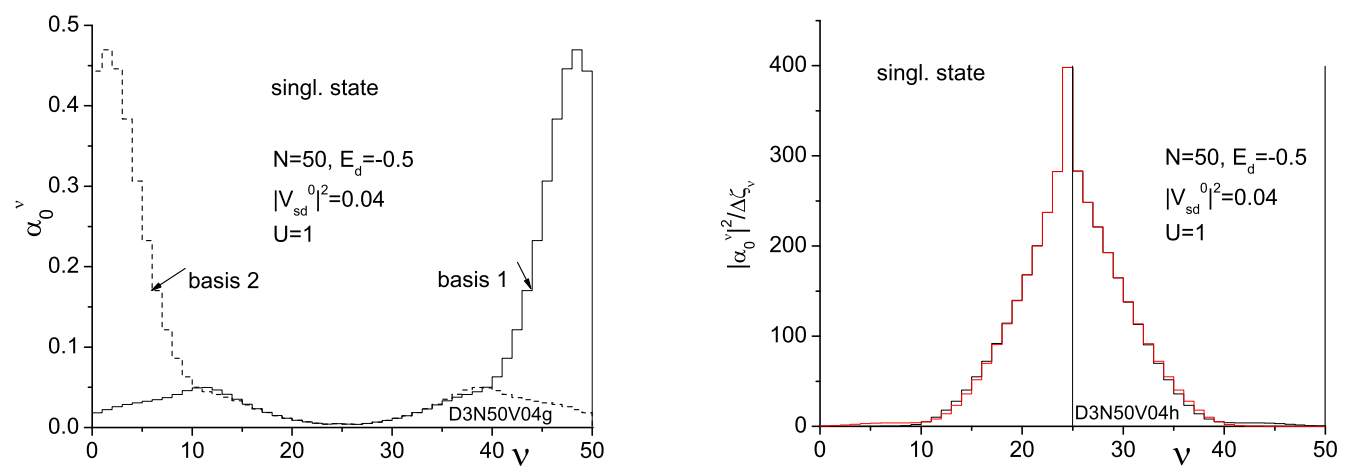

Fig.7a: The coefficients $\alpha_{0 \pm}^{\nu}$ of the FAIR states $a_{0+}^{\dagger}$ and $a_{0-}^{\dagger}$ in terms of the Wilson states $c_{\nu}^{\dagger}$ of the magnetic component $\overline{\Psi_{M S}}$ of the singlet state. Fig.7b: The density distribution of the $p_{\nu}=\left|\alpha_{0 \pm}^{\nu}\right|^{2} / \Delta_{\nu}$ as a function of the cell number $\nu$.

It is interesting to note that the spatial densities of the two FAIR states $a_{0+}^{\dagger}$ and $a_{0-}^{\dagger}$ (after averaging over Friedel oscillations) are identical with an accuracy of four digits. This applies for the magnetic and the singlet state. However, their energy expectation values are essentially opposite equal. The values for $\left\langle a_{0+}^{\dagger} \Phi_{0}|H| a_{0+}^{\dagger} \Phi_{0}\right\rangle$ are rather similar in the magnetic state $(\approx+0.288)$ and the singlet ground state $(+0.2865)$ of the FA impurity.

\subsection{Kondo Impurity}

If one increases the Coulomb potential in the Friedel-Anderson impurity (and lowers the dstate energy $E_{d}$ ) then the coefficients $A_{s, s}$ and $A_{d, d}$ converge towards zero. This is the limit of the Kondo impurity. By means of the Schrieffer-Woulff transformation one can express the s-d-interaction as an exchange interaction of the form $2 J \mathbf{s} \cdot \mathbf{S}$. In this limit the compact ground state $\Psi_{K}$ takes the form

$$
\begin{aligned}
\Psi_{K} & =\left[A_{s, d} a_{0+\uparrow}^{\dagger} d_{\downarrow}^{\dagger}+A_{d, s} d_{\uparrow}^{\dagger} a_{0-\downarrow}^{\dagger}\right] \prod_{i=1}^{n-1} a_{i+\uparrow}^{\dagger} \prod_{j=1}^{n-1} a_{j-\uparrow}^{\dagger} \Phi_{0} \\
& +\left[A_{d, s} a_{0-\uparrow}^{\dagger} d_{\downarrow}^{\dagger}+A_{s, d} d_{\uparrow}^{\dagger} a_{0+\downarrow}^{\dagger}\right] \prod_{i=1}^{n-1} a_{i-\uparrow}^{\dagger} \prod_{j=1}^{n-1} a_{j+\uparrow}^{\dagger} \Phi_{0}
\end{aligned}
$$

Since equ.(6) is a reduced form of equ. (4) the calculation of the integrated net density is analogous to the previous calculation. The Kondo singlet state is composed of two magnetic components represented by the first and the second terms. Note that the effective moment of these magnetic building blocks is less than $1 \mu_{B}$.

We investigate the Kondo ground state for the exchange parameter $J=0.1$. In Fig. 8 the net integrated density is plotted versus the logarithm of the distance $r=2^{l}$ for the magnetic 
component with the d-spin up. One recognizes that a spin polarized "cloud" extends up to a distance of $r=2^{14.7}$ from the Kondo impurity.

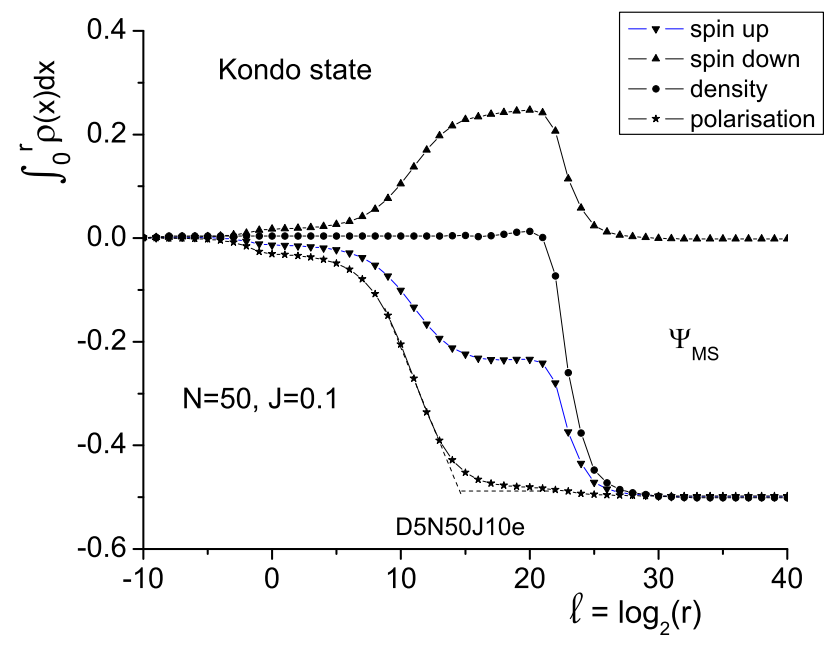

Fig.8: The net integrated density $\int_{0}^{r} \rho(x) d x$ within a distance $r=2^{l}$

from the d-spin up component of the impurity. Shown are the spin up, spin down components as well as the total density and the polarization.

Next we want to study the extent of the Kondo cloud as a function of the Kondo energy. In a recent investigation we obtained for the coupling of $J=0.1$ a relaxed singlet-triplet excitation energy of $\Delta E_{s t}(0.1)=2.35 \times 10^{-5}$. By reducing the coupling to $J=0.08$ we observed an $\Delta E_{s t}(0.08)=1.37 \times 10^{-6}$. The ratio of the two energies is equal to 17.1 .

Fig.9 shows the integrated net s-electron density for a Kondo impurity with $J=0.01$. The two polarization curves (stars) in Fig.8 and Fig.9 are very nicely parallel. Therefore the relative shift of the two curves can be determined very accurately. It is equal to 4.1 in terms of the $\log _{2}$-scale, which corresponds to a length ratio of $2^{4.1}=17.1$. This is a very good agreement with the ratio of 17.1 of the two Kondo energies. Therefore we can confirm that the extent of the screening cloud scales with the Kondo energy. Kondo energy and extension in real space are reciprocal. 


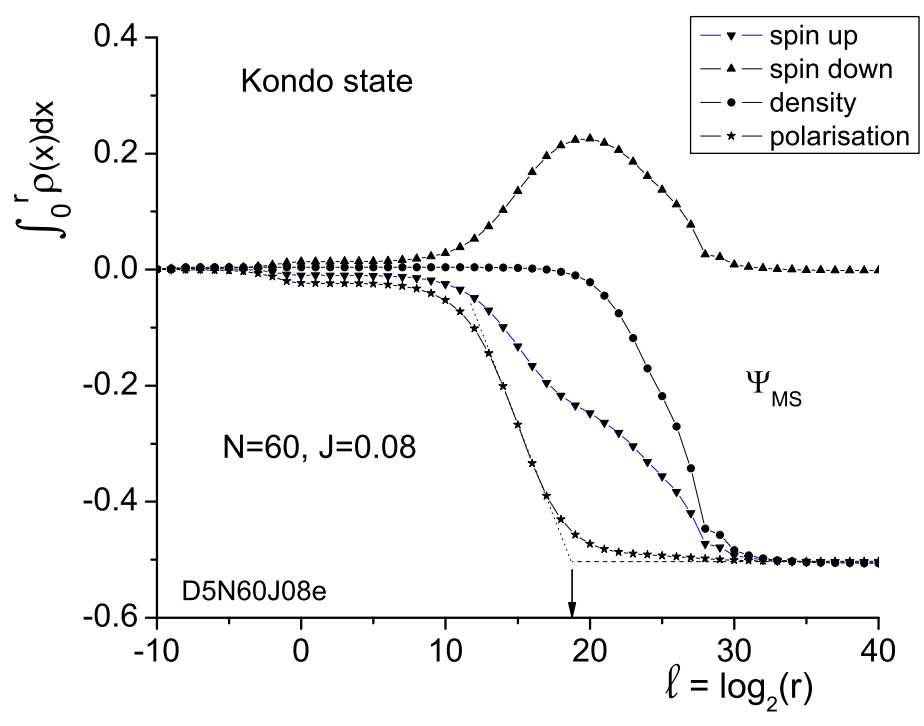

Fig.9: The net integrated density $\int_{0}^{r} \rho(x) d x$ within a distance $r=2^{l}$ from the magnetic component of the impurity. Shown are the spin up, spin down components as well as the total density and the polarization. The polarization extends over a distance of $r=2^{18.8} \approx 4.6 \times 10^{5}\left[\lambda_{F} / 2\right]$.

If in Fig.9 we extrapolate the range of the Kondo cloud as the intersection between the linear decrease and the saturation at the right then we obtain an intersection of $l=18.8$ or a range of $2^{18.8}$ (in units of $\lambda_{F} / 2$ ). In the reduced length units of $\lambda_{F} / 2$ and the energy units of $\varepsilon_{F}$ the Kondo length equ. (1) has the values $\xi_{K}=2 /\left(\pi \varepsilon_{K}\right)$ where $\varepsilon_{K}$ is the Kondo energy in units of $\varepsilon_{F}$. For the parameters of Fig.9 $(J=0.08)$ the Kondo energy has the value $\varepsilon_{K}=\Delta E_{s t}=1.37 \times 10^{-6}$. This yields $\xi_{K}=4.6 \times 10^{5} \approx 2^{18.8}$. The perfect agreement with the numerical extrapolation is probably lucky because the equation (11) for the Kondo length is intended as an order of magnitude.

\section{Conclusion}

A recently developed compact solution for the singlet state of the Friedel-Anderson and the Kondo impurity is used to investigate the old question of a Kondo cloud in the Kondo ground state. Wilson's states with an exponentially decreasing frame of energy cells towards the Fermi level and a linear dispersion relation are expressed in free electron waves integrated over the width of their energy cells. A rotational iteration process (which has been described in previous papers) is used to obtain the optimal ground state. The singlet state of the Friedel-Anderson impurity consists of eight Slater states. They are divided into two groups of four, where each group represents one magnetic component of the singlet state. Two 
criteria yield the same splitting of the eight Slater states: (i) Each group transforms into the other one by inverting all s- and d-spins, (ii) The two groups combine symmetrically in the singlet and anti-symmetrically in the triplet state.

After identifying the magnetic components, the real space wave function is evaluated. Since the spatial range of the Wilson states varies in the range between a Fermi wave length $\lambda_{F}$ and $2^{N / 2} \lambda_{F}(N \approx 50$ is the number of Wilson states used in the evaluation) one has to deal with electron densities which vary be a factor of $2^{25} \approx 3 \times 10^{7}$. Therefore it is favorable to integrate over the net s-electron density from the impurity outwards. The resulting curves yield the spatial range over which the spin densities of the s-electrons contribute to the polarization about the impurity.

If one wants to obtain the polarization about the impurity then one has to calculate the difference of spin up and down s-electron densities with and without the impurity. The simple and exact solution of the Friedel resonance for spinless electrons is used to test the method and interpret the results. It turns out that for each Slater state the behavior of the (integrated) density and polarization can be divided into two regions. For $r>2^{N / 2}$ the system follows the sum rule which is imposed by the number of s- and d-states in the multielectron Slater state. For $r<2^{N / 2}$ one obtains the physical densities and polarizations. The behavior in this range does not change when $N$ is increased. If the d-state in the Friedel resonance is set at the Fermi level then one observes in real space that the range of the s-electron cloud is proportional to $1 /\left|V_{s d}^{0}\right|^{2}$.

For the magnetic state $\Psi_{M S}$ of the Friedel-Anderson impurity one observes essentially no magnetic s-polarization in the vicinity of the d-impurity.

For the magnetic component $\overline{\Psi_{M S}}$ of the singlet state in the Friedel-Anderson impurity one observes an s-polarization cloud which screens the spin (magnetic moment) of the d-electron. The range of this polarization cloud is investigated in detail for the Kondo impurity. The range is inversely proportional to the Kondo energy. The latter was obtained in a previous investigation as the energy difference between the singlet state and the relaxed triplet state energy. The absolute value of the range of the polarization cloud agrees surprisingly well with the Kondo length.

The different screening behavior in the magnetic state $\Psi_{M S}$ and the singlet state $\Psi_{S S}$ is due to subtle differences in the composition of the FAIR states $a_{0+}^{\dagger}$ and $a_{0-}^{\dagger}$. In the singlet state the FAIR states have a much larger weight (of the original basis states $\varphi_{k}$ ) very close to the Fermi energy. It is remarkable that the two FAIR states $a_{0+}^{\dagger}$ and $a_{0-}^{\dagger}$, which are far apart in their energy, have essentially an identical density in real space (after averaging over the Friedel oscillations).

In the present paper a very simple energy band and dispersion relation is used in analogy to Wilson's work. This simplifies the numerical evaluation dramatically. However, the spatial dependence can be evaluated for an arbitrary s-band with an energy-dependent density of states and s-d-interaction. The author generalized the definition of the Wilson states [40] for this case. The numerical work would, however, be much more extensive. (The spin of the impurity is still restricted to $1 / 2$ ).

It should be emphasized that the extent of the Kondo cloud calculated here applies only 
when the mean free path of the conduction electrons is larger than the Kondo length. Also the surface of the host should be at least this distance away from the impurity. This does, however, not mean that for smaller sample size the Kondo effect is suppressed. As long as there are sufficiently many electron states within the Kondo resonance at the Fermi level which couple to the impurity one obtains the full Kondo effect [41.

Finally it should be noted that the extent of the electron density in real space is a detector for a resonance in energy. The spatial extension $\xi$ and the resonance width $\Delta$ are reciprocal and given by the relation $\xi \Delta \approx \hbar v_{F}$. The Wilson states act here as a magnifying glass close to the Fermi energy because their energy width $\Delta_{\nu}$ decreases as $2^{-\nu}$ towards the Fermi level. This is particularly demonstrated for the Friedel impurity in Fig.2. The extent of the net integrated density is only observed when the energy of the d-state lies at the Fermi level. Otherwise it cannot be detected. For example with $E_{d}=-0.5$ the the resolution of the Wilson states is only between 0.5 and 0.25 which is not sufficient to detect the Friedel resonance. One has to split the Wilson states close the resonance to make it visible. Therefore the Wilson states can be used as a quasi-experimental spectroscope.

\section{A The FAIR Method}

The Hamiltonian of the Friedel-Anderson impurity is given in equ. (2). One obtains the mean-field Hamiltonian from equ.(2) by replacing $n_{d+} n_{d_{-}}=>n_{d+}\left\langle n_{d_{-}}\right\rangle+\left\langle n_{d_{+}}\right\rangle n_{d_{-}}$ $-\left\langle n_{d+}\right\rangle\left\langle n_{d_{-}}\right\rangle$. After adjusting $\left\langle n_{d+}\right\rangle$ and $\left\langle n_{d_{-}}\right\rangle$self-consistently one obtains two Friedel resonance Hamiltonians with a spin-dependent energy of the $d_{\sigma}$-state: $E_{d, \sigma}=E_{d}+U\left\langle n_{d,-\sigma}\right\rangle$.

$$
H_{m f}=\sum_{\sigma}\left\{\sum_{\nu=1}^{N} \varepsilon_{\nu} c_{\nu \sigma}^{\dagger} c_{\nu \sigma}+E_{d \sigma} d_{\sigma}^{\dagger} d_{\sigma}+\sum_{\nu=1}^{N} V_{s d}(\nu)\left[d_{\sigma}^{\dagger} c_{\nu \sigma}+c_{\nu \sigma}^{\dagger} d_{\sigma}\right]\right\}
$$

The mean-field wave function is a product of two Friedel ground states for spin up and down $\Psi_{m f}=\Psi_{F \uparrow} \Psi_{F \downarrow}$.

It has been shown [34], 35] that the exact $n$-particle ground state of the Friedel Hamiltonian can be expressed as by the sum of two Slater states, in which either the $d^{\dagger}$-state or a state $a_{0}^{\dagger}$ is multiplied with the same $(n-1)$-s-electron state $\prod_{i=1}^{n-1} a_{i}^{\dagger} \Phi_{0}$ (see equ. (5) ). The state $a_{0}^{\dagger}=\sum_{\nu} \alpha_{0, \nu} c_{\nu}^{\dagger}$ is a localized state whose components $\alpha_{0, \nu}$ can be obtained analytically 35] or by variation [34]. 


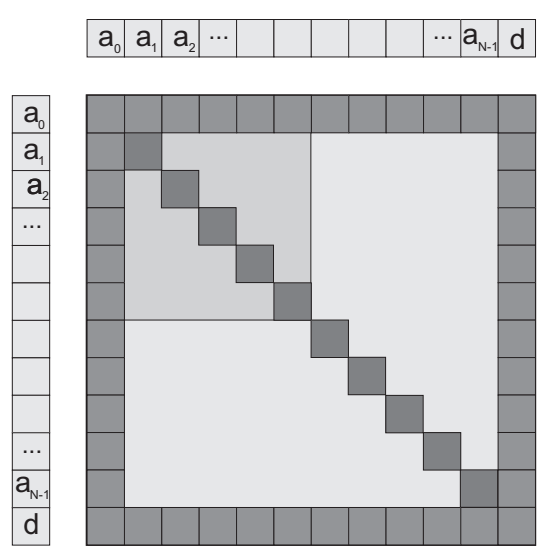

a
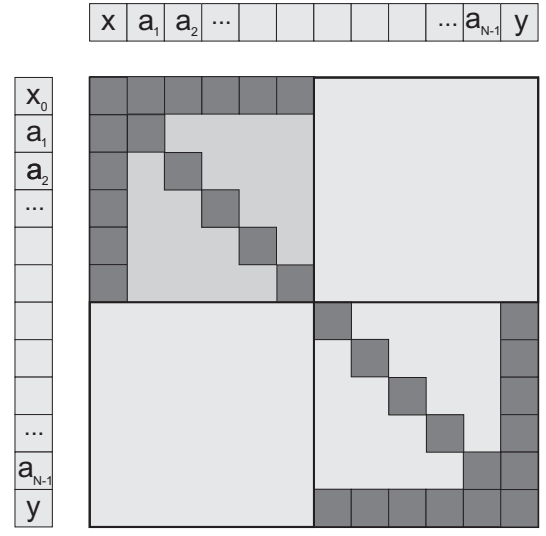

b

Fig.10a: The matrix of the Friedel Hamiltonian in the basis $\left\{a_{i}^{\dagger}\right\}, d^{\dagger}$.

Fig.10b: The two basis vectors $a_{0}^{\dagger}, d^{\dagger}$ are rotated into $x^{\dagger}=\left(A a_{0}^{\dagger}+B d^{\dagger}\right)$ and $y^{\dagger}=\left(B a_{0}^{\dagger}-A d^{\dagger}\right)$. For the optimal $a_{0}^{\dagger}$ the matrix is block-diagonal.

In Fig.10a the matrix of the Friedel Hamiltonian in the basis $\left\{a_{i}^{\dagger}\right\}, d^{\dagger}$ is shown. Only the diagonal elements and the elements along the edges are non-zero. The inner $(N-1) \times$ $(N-1)$-sub-matrix is diagonalized. This way the state $a_{0}^{\dagger}$ determines uniquely the full basis $\left\{a_{i}^{\dagger}\right\}$. One recognizes that the state $a_{0}^{\dagger}$ represents an artificially inserted Friedel resonance state, with similar properties as the state $d^{\dagger}$. Therefore I call $a_{0}^{\dagger}$ a "Friedel artificially inserted resonance" state or FAIR-state. The use of the FAIR-states is at the heart of my approach to the FA- and Kondo impurity problem. Therefore I call this approach the FAIR method.

The structure of the Hamiltonian in Fig.10a can be obtained for an arbitrary state $a_{0}^{\dagger}$. There is, however, one optimal state $a_{0}^{\dagger}$ which yields the exact n-electron eigenstate $\Psi_{F}$ and for which the energy expectation value $\left\langle\Psi_{F}\left|H_{F}\right| \Psi_{F}\right\rangle$ has a minimum. With this state the two basis vectors $a_{0}^{\dagger}, d^{\dagger}$ can be rotated within their plane into the orthogonal states $x^{\dagger}=\left(A a_{0}^{\dagger}+B d^{\dagger}\right)$ and $y^{\dagger}=\left(B a_{0}^{\dagger}-A d^{\dagger}\right)$. For the optimized $a_{0}^{\dagger}$ the resulting Hamiltonian is block-diagonal. This is shown in Fig.10b. The upper left block contains the occupied states. Since there is no matrix-element between the occupied and the empty block the product state $x^{\dagger} a_{1}^{\dagger} a_{2}^{\dagger} . . a_{n-1}^{\dagger} \Phi_{0}=\left(A a_{0}^{\dagger}+B d^{\dagger}\right) \prod_{i=1}^{n-1} a_{i}^{\dagger} \Phi_{0}$ is an eigenstate.

For the two Friedel states in the mean-field wave function I use the form of equ. (5) and obtain for the mean-field solution

$$
\Psi_{m f}=\left[\left(A_{+} a_{0+\uparrow}^{\dagger}+B_{+} d_{\uparrow}^{\dagger}\right) \prod_{i=1}^{n-1} a_{i+\uparrow}^{\dagger}\right]\left[\left(A_{-} a_{0-\downarrow}^{\dagger}+B_{-} d_{\downarrow}^{\dagger}\right) \prod_{i=1}^{n-1} a_{i-\downarrow}^{\dagger}\right] \Phi_{0}
$$


where $\left\{a_{i+}^{\dagger}\right\}$ and $\left\{a_{i-}^{\dagger}\right\}$ are two (different) bases of the $N$-dimensional Hilbert space. This solution can be rewritten as equation (3).

In the mean-field solution $\Psi_{m f}$ the coefficients $A_{\alpha, \beta}$ are restricted by two conditions $A_{ \pm}^{2}+B_{ \pm}^{2}=1$ (here $A_{s, s}=A_{+} A_{-}$etc). Therefore this state does not describe well the correlation effects.

In contrast the state (3) opens a wide playing field for improving the solution: (i) The FAIR states $a_{0+}^{\dagger}$ and $a_{0-}^{\dagger}$ can be individually optimized, each one defining a whole basis $\left\{a_{i \pm}^{\dagger}\right\}$ (which yields a Hamiltonian of the form in Fig.10a) and (ii) the coefficients $A_{\alpha, \beta}$ can be optimized as well fulfilling only the normalization condition $A_{s, s}^{2}+A_{d, s}^{2}+A_{s, d}^{2}+A_{d, d}^{2}=1$. This yields a much better treatment of the correlation effects. The resulting state is denoted as the (potentially) magnetic state $\Psi_{M S}$. The magnetic state $\Psi_{M S}$ has the same form as the mean field solution $\Psi_{m f}$; the only difference is that its components are optimized for the Friedel-Anderson Hamiltonian. The optimization procedure is described in detail in ref. [36].

The magnetic state $\Psi_{M S}$ is used as the building block for the singlet state. It form is given in equ. (4).

\section{B Wilson's states}

Wilson considered an s-band with constant density of states and the Fermi energy in the center of the band. By measuring the energy from the Fermi level and dividing all energies by the Fermi energy Wilson obtained a band ranging from -1 to +1 . To treat the electrons close to the Fermi level at $\zeta=0$ as accurately as possible he divided the energy interval $(-1: 0)$ at energies of $-1 / 2,-1 / 4,-1 / 8, .$. i.e. $\zeta_{\nu}=-1 / 2^{\nu}$. This yield energy cells $\mathfrak{C}_{\nu}$ ranging from $-1 / 2^{\nu}$ to $-1 / 2^{\nu+1}$ with the width $\Delta_{\nu}=\zeta_{\nu+1}-\zeta_{\nu}=1 / 2^{\nu+1}$.

Wilson rearranged the quasi-continuous original electron states $\varphi_{k}(x)$ in such a way that only one state within each cell $\mathfrak{C}_{\nu}$ had a finite interaction with the impurity. Assuming that the interaction of the original electron states $\varphi_{k}(x)$ with the impurity is $k$-independent this interacting state in $\mathfrak{C}_{\nu}$ had the form

$$
\psi_{\nu}(x)=\sum_{\mathfrak{C}_{\nu}} \varphi_{k}(x) / \sqrt{Z_{\nu}}
$$

where $Z_{\nu}$ is the total number of states $\varphi_{k}(x)$ in the cell $\mathfrak{C}_{\nu}\left(Z_{\nu}=Z\left(\zeta_{\nu+1}-\zeta_{\nu}\right) / 2, Z\right.$ is the total number of states in the band). There are $\left(Z_{\nu}-1\right)$ additional linear combinations of the states $\varphi_{k}$ in the cell $\mathfrak{C}_{\nu}$ but they have zero interaction with the impurity and were ignored by Wilson as they will be within this paper.

The interaction strength of the original basis states $\varphi_{k}(x)$ with the d-impurity is assumed to be a constant, $v_{s d}$. Then the interaction between the d-state and the Wilson states $\psi_{\nu}(x)$ is given by $V_{s d}(\nu)=V_{s d}^{0} \sqrt{\left(\zeta_{\nu+1}-\zeta_{\nu}\right) / 2}$ where $\left|V_{s d}^{0}\right|^{2}=\sum_{k}\left|v_{s d}\right|^{2}=\sum_{\nu}\left|V_{s d}(\nu)\right|^{2}$. 


\section{B.1 The wave function of Wilson's states in real space}

For the discussion of the wave functions in real space one has to look a bit closer. We assume a linear dispersion relation between energy and momentum, a constant density of states and a constant amplitude at $x=0$. Then the interaction between the original basis states $\varphi_{k}(x)$ and the d-impurity will be constant for all k-states. These assumption are the same as in Wilson's treatment of the Kondo impurity. We define the wave functions $\varphi_{k}(x)$ is such a way that the results apply for the impurity problem in one, two and three dimensions.

\section{B.1.1 One-dimensional case}

Let us start with the one dimensional problem. Here we have the impurity at the position zero and the conduction electrons are located in the range between 0 and $L$. The wave functions $\varphi_{k}(x)$ have the form $\varphi_{k}(x)=\sqrt{2 / L} \cos (k x)$. There is another set of eigenstates $\overline{\varphi_{k}(x)}=$ $\sqrt{2 / L} \sin (k x)$. These states don't interact with the impurity at the origin. Therefore they don't have any bearing on the impurity problem.

\section{B.1.2 Three-dimensional case}

In three dimensions the free electron states can be expressed as $\varphi_{k}(x)=Y_{l}^{m}(\theta, \phi) j_{l}(k x)$ where $Y_{l}^{m}$ is a spherical harmonics and $l, m$ are the angular momentum and magnetic quantum numbers. $j_{l}(k x)$ is a spherical Bessel function. Its long range behavior is given by $(1 / r \sqrt{2 \pi L}) \sin (k x-l \pi / 2)$. Only the states with the same $l$ as the impurity couple to the impurity. All the other states for different $l$ belong to the group of inert states $\overline{\varphi_{k}(x)}$.

If one calculates the density of the wave function, integrates in the three-dimensional case over the spherical surface $4 \pi r^{2}$ and averages over short range (Friedel) oscillations then one obtains in the

- one-dimensional case: $(2 / L) \overline{\cos ^{2}(k x)}=2 / L$

- three-dimensional case: $\rho_{k}(x)=(2 / L) \overline{\sin ^{2}(k x-l \pi / 2)}=2 / L$

In both cases one obtains essentially the same density. Therefore it is sufficient to use the one-dimensional approach for calculating the density of a Kondo cloud. It is equivalent to the 3-dimensional case integrated over the spherical surface.

\section{B.1.3 The wave functions in one dimension}

While the energy is measured in units of the Fermi energy the momentum will be measured in units of the Fermi wave number. We assume a linear dispersion relation for $0 \leq k \leq 2$ with

$$
\zeta=(k-1)
$$


The (almost) continuous states $\varphi_{k}$ are given as

$$
\varphi_{k}(x)=\sqrt{\frac{2}{L}} \cos (\pi k x)
$$

where $L$ is the length of the one-dimensional box. Since $k$ is measured in units of $k_{F}$ the coordinate $x$ gives the position in units of $\lambda_{F} / 2$ where $\lambda_{F}$ is the Fermi wave number. The boundary condition $\cos (\pi k L)=0$ yields $k=(\lambda+1 / 2) / L$. (The maximal value of $\lambda$ is $2 L$, since $k$ is dimensionless then $L$ is also dimensionless). Therefore we have $Z=2 L$ states in the full band of width 2 .

To obtain the Wilson state we have to sum the states $\varphi_{k}(\pi k x)$ over all states within an energy cell. If the cell ranges from $\left(\zeta_{\nu}: \zeta_{\nu+1}\right)$ corresponding to a $k$-range $\left(1+\zeta_{\nu}\right)<k<$ $\left(1+\zeta_{\nu+1}\right)$ then we represent all the states in this energy interval by

$$
\psi_{\nu}(x)=\frac{1}{\sqrt{\left(\zeta_{\nu+1}-\zeta_{\nu}\right) L}} \sum_{1+\zeta_{\nu}<k<1+\zeta_{\nu+1}} \sqrt{\frac{2}{L}} \cos (\pi k x)
$$

From $Z_{\nu}=L\left(\zeta_{\nu+1}-\zeta_{\nu}\right)$ states we have (according to Wilson) constructed one state $\psi_{\nu}(x)$ which couples to the impurity. The same procedure yields $\left(Z_{\nu}-1\right)$ additional linear combinations of $\varphi_{k}(x)$ which do not couple with the impurity at the origin. We denote these states as $\overline{\overline{\varphi_{k}(x)}}$. They are as inert to the impurity as the states $\overline{\varphi_{k}(x)}$ and will be included in the quasi-vacuum.

The wave function of the state of the states $c_{\nu}^{\dagger}$ has the form for $\nu<N / 2$

$$
\psi_{\nu}(x)=\frac{2 \sqrt{2}}{\sqrt{\left(\zeta_{\nu+1}-\zeta_{\nu}\right)}} \frac{\sin \left(\frac{\pi x\left(\zeta_{\nu+1}-\zeta_{\nu}\right)}{2}\right)}{\pi x} \cos \left(\frac{\pi x\left(2+\zeta_{\nu}+\zeta_{\nu+1}\right)}{2}\right)
$$

For the exponential energy scale this yields for $\nu<N / 2-1$

$$
\psi_{\nu}(x)=2 \sqrt{2^{\nu+2}} \frac{\sin \left(\pi x \frac{1}{2^{\nu+2}}\right)}{\pi x} \cos \left(\pi x\left(1-\frac{3}{2^{\nu+2}}\right)\right)
$$

Similarly one obtains for in the positive energy range

$$
\psi_{N-1-\nu}(x)=2 \sqrt{2^{\nu+2}} \frac{\sin \left(\frac{1}{2^{\nu+2}} \pi x\right)}{\pi x} \cos \left(\pi x\left(1+\frac{3}{2^{\nu+2}}\right)\right)
$$

The two wave functions $\psi_{N / 2-1}$ and $\psi_{N / 2}$ are special because their $k$-range is the same as their neighbors $\psi_{N / 2-2}$ and $\psi_{N / 2+1}$ All four states close to the Fermi level have the same $k$-range of $2^{-N / 2-1}$. One has to pay special attention to this complication. 


\section{B.2 Density of the Wilson states in real space}

The density of the wave function $\psi_{\nu}(x)$ is given by

$$
\left|\psi_{\nu}(x)\right|^{2}=\frac{8}{\left(\zeta_{\nu+1}-\zeta_{\nu}\right)} \frac{\sin ^{2}\left(\frac{\pi x\left(\zeta_{\nu+1}-\zeta_{\nu}\right)}{2}\right)}{(\pi x)^{2}} \cos ^{2}\left(\frac{\pi x\left(2+\zeta_{\nu}+\zeta_{\nu+1}\right)}{2}\right)
$$

The density of a single state $\psi_{\nu}(x)$ is given by the square of the function $\psi_{\nu}(x)$ in equ. (8)). This density has a fast oscillating contribution which yields the Friedel oscillations. We are here interested in the density on a much larger scale and average over the fast oscillation (which has a period of 1 in units of $\lambda_{F} / 2$ ). Then we obtain for the Wilson states

$$
\rho_{\nu}^{0}(x)=\left|\psi_{\nu}(x)\right|^{2}=2^{\nu+3} \frac{\sin ^{2}\left(\pi x \frac{1}{2^{\nu+2}}\right)}{\pi x} d x
$$

In the numerical calculation we will use (most of the time) $N=50$ Wilson states. From equ. (9) one recognizes that the density $\rho_{\nu}^{0}$ of a state $\psi_{\nu}$ lies roughly in the interval between $2^{\nu-2}$ and $2^{\nu+2}$ (in units of $\lambda_{F} / 2$ ). Since for negative energies $\nu$ takes the values from 0 to $(N / 2-1)$ the different wave functions $\psi_{\nu}(x)$ have very different spatial ranges and therefore very different densities, the lowest being of the order of $2^{-25}<3 \times 10^{-8}$. This means that it is not useful to calculate the density as a function of $x$ because this density varies over a range of $2^{25}$. Instead, we use the integrated density, integrated from 0 to $r$.

$$
\begin{aligned}
q_{\nu}^{0}(r) & =\int_{0}^{r}\left|\psi_{\nu}(x)\right|^{2} d x=2^{\nu+3} \int_{0}^{r} \frac{\sin ^{2}\left(\pi x \frac{1}{2^{\nu+2}}\right)}{\pi x} d x \\
& =2 \int_{0}^{\frac{r}{2^{\nu+2}}} \frac{\sin ^{2}(\pi u)}{(\pi u)^{2}} d u
\end{aligned}
$$

One realizes that a single integral yields the integrated density for (almost) all wave function $\psi_{\nu}(x)$. The state $\psi_{0}(\pi x)$ lies roughly in the range between $2^{-2}<r<2^{2}$, i.e. the integrated density $q_{\nu}(r)=\int_{0}^{r}\left|\psi_{0}(\pi x)\right|^{2} d x$ increases in this range from $10 \%$ to $90 \%$. Therefore the states $\psi_{\nu}(x)$ and $\psi_{N-1-\nu}(\pi x)$ lie in the range between $2^{\nu-2}<r<2^{\nu+2}$. For a total of $N=50$ Wilson states the maximum range of the wave functions is roughly $2^{N / 2}=2^{25}$.

We may define as a ruler a linear array $I(s)$ where $s$ is an integer, $(-N / 2 \leq s<N)$ as

$$
I(s)=2 \int_{0}^{2^{s}} \frac{\sin ^{2}(\pi u)}{(\pi u)^{2}} d u
$$

Then the integrated density of the state $\psi_{\nu}$ in the range from 0 to $2^{l}$ is given by

$$
q_{\nu}^{0}\left(2^{l}\right)=\int_{0}^{2^{l}}\left|\psi_{\nu}(x)\right|^{2} d x=2 \int_{0}^{\frac{2^{l}}{2^{\nu+2}}} \frac{\sin ^{2}(\pi u)}{(\pi u)^{2}} d u=I(l-\nu-2)
$$

Then $q_{\nu}^{0}\left(2^{l}\right)$ gives the integrated density on an exponential scale. 


\section{B.2.1 Interference terms in the density}

The Wilson states $\psi_{\nu}(x)$ or $c_{\nu}^{\dagger}$ represent the free electron states in the impurity problem. After the interaction we express the ground state in terms of new states $a_{i}^{\dagger}=\sum_{\nu=0}^{N-1} \alpha_{i}^{\nu} c_{\nu}^{\dagger}$. Its integrated density is given by

$$
\bar{\rho}_{i}\left(2^{l}\right)=\int_{0}^{2^{l}}\left|\sum_{\nu=0}^{N-1} \alpha_{i}^{\nu} \psi_{\nu}(x)\right|^{2} d x
$$

The quadratic terms can be evaluated with the same ruler $I(s)$ as before. But this time one has in addition interference terms $\psi_{\nu}(x) \psi_{\nu+\lambda}(x)$. These terms depend on two parameters, $\nu$ and $\lambda$. So one needs for each $\lambda$ a different ruler. Furthermore the interference terms depend on the sub-bands of $\psi_{\nu}(x)$ and $\psi_{\nu+\lambda}(x)$. If both lie either in the negative energy sub-band $(\nu, \nu+\lambda<N / 2)$ or in the positive sub-band $(\nu, \nu-\lambda \geq N / 2)$ then one obtains one set of rulers $I_{0}(\nu, \lambda)$ and if they lie in opposite sub-bands then one obtains another set of rulers $I_{1}(\nu, \lambda)$. As an example one obtains

$$
I_{0}(s, \lambda)=2 \sqrt{2^{\lambda}} \int_{0}^{2^{s}} \frac{\sin (\pi u) \sin \left(\pi \frac{u}{2^{\lambda}}\right)}{(\pi u)^{2}} \cos \left(3 \pi u\left(1-\frac{1}{2^{\lambda}}\right)\right) d u
$$

For $I_{1}(\nu, \lambda)$ one has to replace the minus sign in the cosine function by a plus sign. Furthermore one has to treat the terms where $\nu+\lambda=N / 2-1$ separately because one state lies at the Fermi level and has a different cell width.

\section{B.2.2 The net integrated density}

If we occupy all Wilson states below the Fermi level then we obtain $\prod_{\nu=0}^{n-1} c_{\nu}^{\dagger} \Phi_{0}$ with $n=N / 2$ and $\Phi_{0}$ the vacuum state. This state is not really the free electron ground state. To obtain the latter we have to occupy the states $\overline{\varphi_{k}(x)}$ and $\overline{\overline{\varphi_{k}(x)}}$. They don't interact with the impurity but they are occupied. Therefore we define as quasi-vacuum $\Phi_{0}^{\prime}$ the state in which all states $\overline{\varphi_{k}(x)}$ and $\overline{\overline{\varphi_{k}(x)}}$ with $k<k_{F}=1$ are occupied. Then the ground state is $\Psi_{0}=$ $\prod_{\nu=0}^{n-1} c_{\nu}^{\dagger} \Phi_{0}^{\prime}$. This state has a constant electron density in real space.

In the presence of the impurity the new ground state $\Psi_{\text {new }}=\prod_{i=0}^{n-1} a_{i}^{\dagger} \Phi_{0}^{\prime}$ must also contain this quasi vacuum, i.e., the non-interacting states must be occupied up to the Fermi level. Since the inert states are occupied in $\Psi_{0}$ and $\Psi_{\text {new }}$ they cancel out when one calculates the change in the electron density. The net density of the new state $\Psi_{n e w}$ is the difference between $\rho\left(\Psi_{\text {new }}\right)$ and $\rho\left(\Psi_{0}\right)$. Since the inert states cancel out one can ignore their existence during this calculation. 


\section{References}

[1] J.Friedel, Philos.Mag. 43, 153 (1952); Adv.Phys. 3, 446 (1954); Philos.Mag.Suppl. 7, 446 (1954); Can.J.Phys. 34, 1190 (1956); Nuovo Cimento Suppl. 7, 287 (1958); J. Phys.Radium 19, 38 (1958)

[2] P.W.Anderson, Phys.Rev. 124, 41 (1961)

[3] J.Kondo, Prog.Theor.Phys. 32, 37 (1964)

[4] K.Yosida, Phys.Rev. 147, 223 (1966)

[5] C.M.Varma and Y.Yafet, Phys.Rev. B13, 2950 (1976)

[6] K.Schoenhammer, Phys.Rev. B 13, 4336 (1976)

[7] M.D.Daybell, and W.A.Steyert, Rev. Mod. Phys. 40, 380 (1968)

[8] A.J.Heeger, in Solid State Physics, ed. by F.Seitz, D.Turnbull, and H.Ehrenreich (Academic, New York, 1969), Vol 23, p284

[9] M.B.Maple, in "Magnetism", edited by G.T.Rado and H.Suhl (Academic, New York, 1973), Vol.V, p.289

[10] P.W.Anderson, Rev.Mod.Phys. 50, 191 (1978)

[11] G.Gruener and A.Zavadowski, Prog.Low Temp.Phys. 7B, 591 (1978)

[12] P.Coleman, J. Magn.Magn.Mat. 47, 323 (1985)

[13] A.C.Hewson, The Kondo problem to heavy Fermions, Cambridge University Press, 1993

[14] P.W.Anderson, J.Phys. C3, 2436 (1970) 
[15] K.G.Wilson, Rev.Mod.Phys. 47, 773 (1975)

[16] H.O.Frota and L.N.Oliveira, Phys.Rev. B33, 7871 (1986)

[17] H.R.Krishna-murthy, J.W.Wilkins, and K.G.Wilson, Phys.Rev. B 21, 1003 (1980)

[18] H.R.Krishna-murthy, J.W.Wilkins and K.G.Wilson, Phys.Rev. B 21, 1044 (1980)

[19] P.Nozieres, J.Low Temp.Phys. 17, 31 (1974)

[20] P.Nozieres, Ann.Phys. (Paris) 10, 19 (1985)

[21] D.M.Newns and N.Read, Adv. in Phys. 36, 799 (1987)

[22] O.Gunnarsson and K.Schoenhammer, Phys.Rev. B28, 4315 (1983)

[23] N.E.Bickers, Rev.Mod.Phys. 59, 845 (1987)

[24] P.B.Wiegmann, in Quantum Theory of Solids, edited by I.M.Lifshits (MIR Publishers, Moscow, 1982), p. 238

[25] N.Andrei, K.Furuya, and J.H.Lowenstein, Rev.Mod.Phys. 55, 331 (1983)

[26] P.Schlottmann, Phys.Reports 181, 1 (1989)

[27] P.Nozieres, and A.Blandin, J.de Physique 41193 (1980)

[28] J.B.Boyce, and C.P.Slichter, Phys. Rev. Lett. 32, 61 (1974)

[29] I.Affleck, and P.Simon, Phys.Rev.Lett. 86, 2854 (2001)

[30] I.Affleck, arXiv:cond-mat/0111321 (2001) 
[31] R.G.Pereira, N.Laflorencie, I.Affleck, and B.I.Halperin, arXiv:cond-mat/0612635 (2007)

[32] L.Borda, Phys.Rev. B 75, 041307(R) (2007)

[33] J.Simonin, arXiv:0708.3604 (2007)

[34] G.Bergmann, Z.Physik B102, 381 (1997)

[35] G.Bergmann, Eur.Phys.J.B2, 233 (1998)

[36] G.Bergmann, Phys.Rev. B 73, 092418 (2006)

[37] G.Bergmann, Phys.Rev. B 74, 144420 (2006)

[38] G.Bergmann and L.Zhang, Phys.Rev. B 76, 064401 (2007)

[39] O.Gunnarsson and K.Schoenhammer, Phys.Rev. B31, 4815 (1985)

[40] G.Bergmann, cond-mat/061020, subm. to Phys.Rev.B

[41] G.Bergmann, Phys.Rev.Lett. 67, 2545 (1991) 